\title{
Numa Urbe Genderizada: vivências dos espaços
}

\author{
In a Gendered City: living the spaces
}

\author{
Joana Miranda \\ Universidade Aberta \\ Centro de Estudos das Migrações e das Relações Interculturais (CEMRI) - Portugal \\ joana@uab.pt
}

\section{Resumo}

A cidade foi sendo perspetivada como um lugar masculino em que as mulheres (e, em particular, as mulheres imigrantes) não eram cidadãs plenas no sentido em que não adquiriram o acesso integral e livre às ruas e sobreviveram nos interstícios da cidade. Apesar de todas as conquistas das mulheres e do aparente cosmopolitismo das cidades europeias, as cidades continuam a ser espaços genderizados, espaços de conflito e de discriminação, contextos plenos de ameaças e interdições. Neste artigo apresentaremos algumas reflexões sobre a forma como as mulheres imigrantes brasileiras, cabo-verdianas e ucranianas se relacionam com o espaço - cidade de Lisboa - com base nos dados do projeto de investigação da autora financiado pelo Observatório da Imigração 'Mulheres imigrantes em Portugal. Memórias, dificuldades de integração e projetos de vida'.

Palavras-chave: Cidade; Espaço Urbano; Mulheres Imigrantes; Género.

\begin{abstract}
The city had been seen as a masculine place in which women (and in particular immigrant women) weren't full citizens in the sense that they didn't acquire the integral and free access to the streets and they survived in the city' interstices. Although all women's conquests and the apparent cosmopolitanism of European cities, the cities continue to be gendered spaces, spaces of conflict and discrimination, contexts full of threats and interdictions. In this article, based on the data obtained with the project sponsored by ACIDI 'Immigrant women in Portugal. Memories, integration problems and life projects', we present some reflections related to the way immigrant women: Brazilian, Cape Verdean and Ukrainian women relate to the space - the city of Lisbon.
\end{abstract}

Key-words: City; Urban Space; Immigrant Women; Gender. 


\section{Contextualização teórica}

The rhizome is not nomadic, it roots itself, even in the ai Edouard Glissant

\section{O espaço da cidade}

Tradicionalmente o lugar é perspetivado como uma localização física particular caracterizada por um conjunto fixo de características sociais (WOMEN AND GEOGRAPHY GROUP, 1997). Na última década verificou-se um interesse crescente em reconcetualizar o espaço e o lugar, bem como as inter-relações entre espaço, lugar, identidade e vidas materiais (vide, p. ex., JACKSON \& PENROSE, 1993; KEITH \& PILE, 1993; MASSEY, 1998). Diversos trabalhos, nomeadamente o de Massey (1993, 1998), questionaram a tradicional perspetivação do lugar como um local (place) definido e fixo, articulando-o com as relações sociais, contestando o isomorfismo assumido do espaço, lugar e cultura. De facto, se analisarmos as relações sociais desde as mais locais até as mais globais, verificamos que a identidade não está encerrada num lugar fixo e delimitado (MASSEY \& DELTON, 1993) e que as diferentes identidades sociais (de género, migrante) são construídas através de uma complexa rede de relações sociais, no contexto de espaços e lugares sobrepostos. No entanto, também não nos parece adequada a reificação desenraizada como uma figura paradigmática da vida pós-moderna.

As identidades culturais são desterritorializadas e reterritorializadas, utilizando termos criados por Giles Deleuze e por Felix Guatarri (1980) na sua tentativa de teorização da constituição do território através do movimento.

Numa entrevista a Claire Parnet, Gilles Deleuze declarou que "não existe desterritorialização sem um esforço de reterritorialização" (p. x) (BOUTANG e PAMAR, 1995). Mas a reterritorialização não deve ser confundida com o retorno a uma territorialidade mais primitiva ou mais antiga. De uma forma semelhante à teoria da tradução de Walter Benjamin ou ao conceito de 'hibridez' de Homi Bhabha (1990), Deleuze e Guatarri (1980) sugerem que os elementos se reterritorializam uns nos outros. Mas cada elemento mantem alguma da sua territorialidade e a sua territorialidade combinada produz uma nova territorialidade.

Centremos a nossa reflexão sobre o espaço urbano. Os espaços (spaces) e os lugares (places) não são naturais, neutrais ou entidades pré-definidas, são, antes, construídos (JACKSON e PENROSE, 1993), são arenas com significado político. Keith e Pile (1993) salientam: "Space cannot be dealt with as if it was merely a passive, abstract arena on which things happen" (p. 2).

As conceções que temos das diferentes cidades resultam dos discursos, das narrativas, das histórias construídas sobre essas mesmas cidades e não são mais do que interpretações dessas cidades. Num dado momento podem coexistir discursos concorrentes sobre uma mesma cidade, podendo fatores de ordem diversa contribuir para que um discurso torne-se dominante e para que os outros discursos sejam silenciados. Diversos autores salientam o caráter construído dos discursos sobre diferentes cidades situadas um pouco por todo o mundo. A título exemplificativo, Herbert (2007) salienta a construção de Leicester como cidade cosmopolita em que a comunidade asiática assume grande significado, Eade (2000) analisa como Londres foi promovida como espaço de melting-pot de sucesso de diferentes grupos de imigrantes; Walton (2001) refere-se ao discurso sobre Monterey. Nesta linha é também possível considerar que, para além do discurso de uma Lisboa multicultural e intercultural, aberta ao outro, existem as vivências das populações migrantes que nos 
dão perspetivas outras da cidade e que testemunham as décalages tantas vezes existentes entre os discursos das sociedades recetoras (grupos dominantes) e as práticas dos imigrantes (grupos dominados).

Enfatizar o carácter construído dos discursos sobre as cidades não significa que esses discursos sejam inteiramente falsos, apenas significa que constituem discursos (entre diversos outros discursos possíveis) e que as realidades das populações imigrantes que vivem nessas cidades são, por vezes, bem distintas do discurso dominante e que são também, inevitavelmente, mais polifacetadas.

Não sendo neutro (haverá espaços que sejam integralmente neutros?), o espaço urbano é um espaço em que se jogam relações de poder, estratégias de dominação de uns grupos em relação a outros e, apesar das mudanças sociais que vão ocorrendo na forma e na intensidade dessas relações, persiste uma continuidade opressiva. Eventos culturais em que ocorre o controle simbólico das ruas e que invertem temporariamente a estrutura do poder urbano permitem a quebra simbólica dessa continuidade. É, por exemplo, o caso do The West Indian Labor Day Parade em Brooklyn, Nova Iorque (KASINITZ, 1992); da Halloween Parade em Greenwich Village, Nova Iorque (KUGELMASS, 1994) ou de Las Fallas em Valência (LAWRENCE, 1992).

Autores como Jackson e Penrose (1993) salientam a racialização dos lugares no espaço urbano. Diversos estudos analisam a forma como os grupos dominantes construíram e organizaram a diferença e regularam 'os outros', os diferentes, no contexto de cidades multi-étnicas (CROSS \& KEITH, 1993; GRILLO, 2000). Mas o que nos interessa não é apenas a construção do espaço em função de variáveis nacionais e étnicas, mas também da variável género ou, se preferirmos, a genderização do espaço.

Interessa-nos focalizar a análise na comunidade/s imigrante/s na cidade e a sua relação com o espaço. A noção de comunidade remete para uma história comum, experiência ou cultura de um grupo (aquilo que Anne-Marie Fortier define por 'migrant belongings'), mas está também ligada a lugares, imaginários ou reais (GUPTA \& FERGUSON, 1992).

Para Probyn (1993), o conceito de pertenças (belongings) seria preferível ao de identidade porque deslocaria a identidade do seu estatuto fundacional, permitindo captar o desejo das pessoas de estabelecerem algum tipo de ligação, seja a lugares, a pessoas ou a modos de ser, bem como a forma como os indivíduos e os grupos se esforçam por pertencer, por fazer parte de um todo, como se desejam tornar, num processo que está marcado por ânsia.

Considerando o mundo social como uma superfície, Probyn (1996) enfatiza o caráter estremecido (quivery) da identidade. A identidade como um limiar (threshold), isto é, uma localização (location) que, por definição, enquadra a passagem de um espaço para outro, a identidade como uma transição, produzindo-se sempre através dos processos combinados de ser e de se tornar. Enquanto que Probyn adota uma perspetiva Deleuziana que enfatiza o movimento (o seu livro Sexing the Self está repleto de várias formas de locomoção: comboios, aviões, cavalos), Fortier (2000) perspetiva as pertenças migrantes como sendo constituídas simultaneamente por movimento e ligação. $\mathrm{Na}$ formação da pertença, a identidade surge como uma posicionalidade momentânea (HALL, 1996) que já se tornou (already becoming). Há que procurar os pontos momentâneos de ligação ('pontos de sutura' nas palavras de Stuart Mill, 1990), bem como os pontos de partida. Como Caren Kaplan (1996) assinala, até as teorias de rizomas nómadas incluem nodos, esses pontos de interceção de movimentos de 'linhas de luta'1. 
Certeau (1984) salienta que as atividades corporais na cidade são parte de um processo de apropriação do espaço e de territorialização. Os indivíduos caminham (a forma mais elementar de vida na cidade) pelas ruas, são 'walkers' cujos corpos seguem as linhas de um 'texto' urbano. A prática do caminhar possibilita dar sentido ao espaço e as repetições dos actos são, de facto, uma forma de ultrapassar a alienação (LEACH, 2002). O sentido de pertença e de ligação que se desenvolvem com o tempo são estabelecidos com base no conhecimento adquirido, na memória e na experiência repetida de uso, especialmente o caminhar.

A pertença surge como uma 'teoria da territorialização', o espaço é um lugar prático. As ruas geometricamente definidas pelo planeamento urbano transformam-se num espaço pelos caminhantes e, num certo sentido, ganham vida. Essas práticas repetidas incluem-se no que Vicki Bell (1999) define como 'performatividade e pertença', entendendo a performatividade como a replicação e a repetição de certos desempenhos que estão associados com práticas ritualistas com que as comunidades colonizam vários territórios.

\section{A Genderização do Espaço Urbano}

No início do século $\mathrm{XX}$ as mulheres adquiriram uma crescente mobilidade. Segundo Parsons (2000) as ruas da cidade proporcionaram às mulheres a possibilidade de escaparem do escrutínio da esfera doméstica. De facto, muitas das preocupações com a mobilidade das mulheres na cidade tiveram por base a dificuldade de controlar os movimentos das mulheres em multidões vastas e anónimas (MASSEY, 1998; PARSONS, 2000). Apesar dessa liberdade estar limitada e sujeita à manipulação dos empregadores, a sua importância para a emancipação não pode ser descurada
(PARSONS, 2000).

Wilson (1991) salienta que o ambiente urbano possibilita um determinado grau de autonomia, anonimato e liberdade que as mulheres dificilmente conseguem experimentar nos subúrbios ou nas cidades pequenas. Wekerle (1984) refere que, ao contrário dos subúrbios, as cidades suportam os vários papéis das mulheres $\mathrm{e}$ as académicas feministas enfatizam as escolhas oferecidas às mulheres na cidade (DARKE, 1996; WILSON, 1991).

Mas Wilson (1991) acrescenta que a negociação dos espaços urbanos pelas mulheres continua a ser limitada por questões de segurança e respeitabilidade. A par do medo de serem ameaçadas ou mesmo atacadas por estranhos, medo parcialmente justificado por uma natural maior fragilidade física comparativamente aos homens, imagens poderosas da 'mulher desviante', da 'street walker', da 'mulher das ruas', noutras palavras, da prostituta, limitam a liberdade de movimentos das mulheres 'respeitáveis' na cidade, quer em culturas ocidentais quer não ocidentais. Assim, a cidade revela-se um espaço simultaneamente libertador e ameaçador. A mobilidade espacial das mulheres continua condicionada pelas significações culturais simbólicas do espaço, pelos espaços de pertença e de não-pertença, permitidos ou não permitidos (FENSTER, 1998). Nestas condições, o 'direito à cidade' a que Lefebvre se refere, direito ao uso de espaços públicos, acaba por não encontrar uma concretização.

Massey (1998) refere-se ao dualismo entre homens e mulheres que atravessa a conceção do espaço. Existe uma forte associação entre o feminino e o doméstico e o polo da dicotomia local/feminino é desvalorizado em relação ao polo global/masculino. A mulher/mãe é associada a casa e a um lugar fixo. A mobilidade das mulheres, a sua 'transgressão' do local pode ser perspetivada 
como uma ameaça à genderização patriarcal do espaço (MASSEY, 1998). Mas também a casa pode ser um espaço contestado e um espaço de violência doméstica, existem situações em que a cidade se pode transformar no espaço privado que a casa não é, transformado em lugar de perigo, de ameaça em que o agressor não é alguém desconhecido mas alguém com quem se partilha a intimidade.

Wilson (1991) refere que as mulheres (tal como os pobres e outras minorias) vivem nos 'interstícios' da cidade, Hayden (1995) à invisibilidade dos contributos das mulheres nos monumentos de Los Angeles. Como Fenster (2005) refere algumas mulheres percecionam os parques como áreas masculinas hostis, áreas 'conquistadas' pelos homens. Quais são, pois, os lugares seguros, os espaços que respondem ao direito de uso? Mesmo em cidades aparentemente seguras como Lisboa existem espaços pouco seguros para as mulheres. Como se apropriam as mulheres imigrantes do espaço e como se articula essa apropriação com a incorporação (embody) da sua terra natal?

Mas não se coloca apenas a questão da mobilidade das mulheres imigrantes no espaço, mas o direito das mulheres a tomarem decisões relativas à produção dos espaços urbanos. Young (1998) refere que as mulheres participam menos do que os homens nestas decisões e uma década mais tarde, essa situação continua a verificar-se. Quantas mulheres, que percentagem de mulheres e de mulheres imigrantes, em particular, participam, a nível nacional e local, nas decisões políticas sobre organização do espaço urbano, quantas fazem parte de associações de imigrantes e nelas desempenham papéis de liderança, exercendo o seu poder?

Young (1998) utiliza o termo 'paradoxo da democracia' para se referir aos poderes sociais que tornam certas pessoas mais poderosas do que outras. O poder de voto e de escolha aumenta o sentimento de pertença e poderemos facilmente pensar que, se as mulheres imigrantes tivessem maior poder decisional a este nível, veriam reforçados os seus sentimentos de pertença.

Diversos estudos relacionam género e espaços. Fine (1992) estudou os protestos das mulheres contra o seu silenciamento nas escolas públicas secundárias, Katz (1993) a sua exclusão dos locais de aquisição do conhecimento no Sudão e em Nova Iorque e Macleod (1991), a forma como são controladas por hegemonias tradicionais e ocidentais no Cairo.

Procurando alicerçar a reflexão teórica num contexto de terreno, analisarei, em seguida, alguns aspetos resultantes do projeto financiado pelo Observatório da Imigração: 'Mulheres imigrantes em Portugal. Memórias, dificuldades de integração e projetos de vida'. Este projeto procurou articular as variáveis migrações e género, articulação essa que tem sido pouco frequente no domínio das migrações em Portugal. Apesar da variável espaço não ter sido uma das variáveis privilegiadas na análise procuraremos analisar alguns dos resultados que com ela se relacionam.

\section{Histórias de Vida de Mulheres Imigrantes}

As histórias orais ou narrativas de vida apenas revelam o que as pessoas desejam contar. $\mathrm{O}$ que é contado não é, necessariamente, o que é recordado: 'memory is inherently revisionist' (GARDNER, 1999) e compomos as nossas memórias para dar sentido às nossas vidas passadas e presentes (THOMSON, 1998). As narrativas pessoais são frequentemente inconsistentes e confusas, raramente seguindo qualquer tipo de ordem cronológica (CHAMBERLAIN, 1997). Mas tal não questiona $\mathrm{o}$ seu significado $\mathrm{e}$ relevância. De facto, os erros e as 
contradições da memória permitem-nos compreender os significados subjetivos da experiência histórica (THOMSON, 1998). O nosso objetivo no estudo não foi chegar a uma recordação precisa do passado, mas à forma como as mulheres explicam, descrevem e dão sentido às decisões que tomaram e às vidas que escolheram. Apesar de cada história ser única e pessoal e da questão do espaço não estar particularmente presente nas questões colocadas surgiam referências recorrentes ao espaço, ao lugar e à mobilidade.

As histórias orais revelam relações ativas com o espaço e construções desse mesmo espaço, no caso do nosso estudo construções realizadas por mulheres imigrantes brasileiras, cabo-verdianas e ucranianas residentes na área metropolitana de Lisboa e exercendo funções indiferenciadas.

Massey (1998) relaciona a nostalgia com um lugar, mas como Ryan (2003) salienta, a nostalgia está também relacionada com o movimento. E o primeiro movimento destas mulheres foi a viagem para Portugal.

\section{Metodologia}

Seguimos uma metodologia qualitativa em que realizámos entrevistas semi-diretivas a vinte e quatro mulheres imigrantes em Portugal: oito mulheres brasileiras, oito mulheres ucranianas e oito mulheres caboverdianas. Essas comunidades são atualmente as de maior dimensão em Portugal. As mulheres selecionadas não pretendem dar conta da totalidade das mulheres imigrantes residentes em Portugal, mas antes dar conta da multiplicidade de situações em que se encontram. Os resultados foram analisados recorrendo a análise de conteúdo. Analisaram-se resultados caso a caso, por nacionalidade e o conjunto total das mulheres. Os resultados que apresentamos neste artigo correspondem a uma pequena parte dos resultados encontrados que podem ser localizados na publicação do projeto e que tem o mesmo título do referido.

\section{Resultados}

\section{A viagem}

Porque vieram, afinal? Curiosamente, na idade das diásporas (BAUMAN: 2007) e no mundo globalizado e desterritorializado: 'a viagem' (de avião ou de autocarro, solitária ou na companhia de outros) não foi mais um trajeto no seio de uma tessitura de outros trajetos.

A metáfora de Rosa Braidotti (1994) de mulheres movendo-se entre diferentes mundos, linguagens, trabalhos e lugares sem estarem ligadas a uma localização fixa revelase atrativa porque transmite a representação de mulheres 'on the move', empoderadas, dotadas de estratégias para evitar o racismo e o sexismo, mas não parece mais ser que uma metáfora. Celebrar a mobilidade só é possível em determinadas condições ou para determinadas mulheres, mulheres qualificadas cujas formações académicas e as competências profissionais as dotam de um certo grau de independência e o investimento num percurso escolar revela-se um requisito para a mobilidade e para o transnacionalismo bem sucedido.

A viagem para Portugal foi, na grande maioria dos casos, 'a viagem', o ponto de viragem, a data que marcou a separação entre a vida que se tinha antes e a vida que se passou a ter. A grande maioria das mulheres nunca tinha saído do seu país, da sua região e, no caso das cabo-verdianas, das suas ilhas de origem. As datas de chegada são genericamente recordadas com precisão mesmo quando muito distantes no tempo: dia, mês e ano e são enunciados com a solenidade com que se declamam as grandes datas, pelo valor simbólico e, também, naturalmente, 
pelo valor instrumental, uma vez que o tempo de permanência possibilita a autorização de residência e, em consequência, a aquisição de direitos. De resto, são exceção às mulheres que alimentam projetos de migração para outros países e os projetos de vida circunscrevem-se a dois grandes subtipos: os projetos de retorno ao país de origem (mais frequentes entre as mulheres cabo-verdianas) e os de permanência em Portugal (para além de projetos de compra de casa, de melhores ordenados e de estar mais próximo de quem se ama).

\section{O Primeiro Contacto com a Cidade}

Nos estudos de Gardner (1999) e de Chamberlain (1997) os entrevistados falam acerca dos seus ambientes sociais imediatos e das suas experiências ao atravessar espaços hostis da cidade, a forma como conseguem negociar a cidade hostil e anónima e transformar um espaço estranho numa 'homeplace'. As entrevistas do estudo revelam esta progressiva negociação de um espaço inicialmente hostil ou, pelo menos, não familiar e a construção de uma 'homeplace'.

$\mathrm{O}$ primeiro contacto com a cidade de Lisboa induz surpresa: centros comerciais de grandes dimensões, a pedra das calçadas que dificulta o caminhar de saltos altos das ucranianas, o grande número de pessoas, a grande concentração de edifícios, a ausência do verde dos jardins da Ucrânia ou da paisagem árida e das praias de Cabo Verde. A paisagem surpreende e assusta. Para além das diferenças na paisagem relativamente à paisagem do país de origem sente-se a estranheza dos hábitos, das maneiras de ser e de sentir dos nacionais, as diferenças de clima e de língua. A cidade surge também como diferente daquela que tinham imaginado a partir dos folhetos turísticos ou que tinham construído com base nos discursos dos amigos e familiares que já tinham estado em
Lisboa.

Independentemente da estranheza do espaço físico importa também compreender que as sensações de desenraizamento que marcam os primeiros tempos na cidade decorrem em grande parte desses espaços não serem habitados pelas figuras habituais dos afetos.

\section{Memórias do País de Origem}

As mulheres falam do país de origem tendo por quadro de referência as suas famílias: mães, pais, irmãos e irmãs. O país de origem é descrito através das suas experiências pessoais, familiares e comunitárias. A família é o lugar privilegiado e o suporte da memória (FORTIER, 2000). O país foi o local em que se nasceu, onde se cresceu e se desenvolveram as primeiras relações amorosas, onde se envolveram em projetos de conjugalidade, onde se constituiu família, onde os filhos nasceram, onde, para algumas, os pais morreram. São as memórias dos tempos de escola, dos colegas de escola, dos rituais e práticas, das danças, músicas e comidas, das rezas, dos pequenos traumas de infância. A apreciação mais ou menos negativa das suas vidas em Portugal está sempre dependente dessas mesmas memórias e resulta da comparação com o grau em que esse passado foi gratificante. Assim, existe uma tendência para as mulheres mais ligadas ao passado e que mais valorizam as memórias terem mais projetos de retorno aos países de origem e para as mulheres menos ligadas ao passado e que menos valorizam as memórias terem menos projetos de retorno aos países de origem.

\section{Espaços Permitidos}

Apesar de em teoria a cidade abrir um mundo de possibilidades de descoberta, os espaços percorridos circunscrevem-se aos 
locais de trabalho, aos percursos para o trabalho, às igrejas, no caso das mulheres praticantes, às casas dos familiares e amigos e a locais intrinsecamente relacionados com as satisfações das necessidades do quotidiano como supermercados, centros de saúde, entre outros. São pouco significativos os espaços de lazer referidos: praias, cafés, cinemas e pouco mais. A maioria das mulheres conhece mal o país e apenas visitou locais muito turísticos, nomeadamente, Serra da Estrela, Algarve e Fátima. Assim, o conhecimento do país é muito limitado e circunscreve-se aos locais que possibilitam a satisfação das necessidades básicas. A maioria das mulheres que regressa aos países de origem tem uma imagem superficial e simplificada do país em que viveu e trabalhou, muitas vezes durante muitos anos. Tal explica-se na generalidade dos casos por horários longos de trabalho, por tarefas duras, por acumulação frequente de mais do que um trabalho com consequências a nível físico. Muitas mulheres aproveitam os fins-de-semana para tratarem das suas próprias casas ou simplesmente para descansar.

\section{Negociando a Cidade}

Por entre percursos marcados por conflitos internos (e alguns externos com os nacionais e com os imigrantes de origens diferentes) essas mulheres vão-se embrenhando em processos de mudança, afirmam-se, diferenciam-se, escondem-se, negoceiam, antagonizam-se, tornam-se cúmplices, jogam, reconstroem-se, desenvolvem estratégias identitárias (no sentido em que Camilleri (1991) se refere a estratégias identitárias) de estar e de ser que se revelam diferentes de mulher para mulher.

Com o tempo as mulheres transformam-se, vão-se descobrindo diferentes das que outrora partiram dos seus países. Não são quem eram, não são como os compatriotas que deixaram, imutáveis, no país de origem, como peças de xadrez num tabuleiro imóvel, mas não são, também, semelhantes as cidadãs do seu novo território. Quando, nas férias, regressam ao país natal descobrem-se estranhas na sua terra e estranhas também nesta nova terra, estrangeiras: 'Em Portugal, como já disse, sinto-me ucraniana, mas na Ucrânia sinto-me estrangeira'. Quem são elas, afinal?

Que estratégias desenvolvem para lidar com a situação? Algumas se refugiam no passado e intensificam a importância das raízes, mistificando-o, imbuindo-o de saudade e de nostalgia. São as memórias das avós, das mães, dos colegas, dos amigos de rua, dos tempos de escola, da geografia dos locais: 'Gosto da ilha como espaço, tem coisas muito boas (...) nunca tinha saído da ilha, ela é grande'.

Outras mulheres, pelo contrário, distanciam-se do passado, separam-se dele e procuram tornar-se cidadãs do mundo: 'Eu não pego no passado, porque é museu que guarda passado, porque tudo o que eu fiz está feito, está feito. Eu não tenho arrependimento de nada e procuro viver o hoje'.

Para muitas o passado é já um lugar distante que corre o risco de se dissipar, um cenário trémulo e provisório que são os outros que ajudam a compor: 'Recordações que eu tenho de Cabo Verde é muito poucochinho. Só me lembro de uma rocha e de uma praia! De resto não me lembro de mais nada. Com três anos, também (...). Mas ouço falar, de pessoas, familiares que (...) vieram mais tarde e que falam sobre isso'.

Para outras, ainda, o passado é o lugar onde se nasceu, mas não é mais do que isso: a recordação de um lugar, de um ponto geográfico: 'Cabo Verde foi onde eu nasci. Mas não passa muito disso'.

Com o tempo muitas das dificuldades iniciais são superadas e as mulheres conquistam melhores condições de vida que estão muito associadas aos processos de 
regularização e de aquisição de residência. A nível habitacional, verifica-se uma melhoria generalizada. As duas mulheres que habitavam inicialmente em barracas deixaram de viver em barracas. As que viviam em casas mais degradadas e com menos espaço adquirem casas com mais espaço e melhores condições, conseguem casas com rendas menos elevadas e condições similares, deixam de partilhar as casas com os amigos e com os familiares mais afastados e passam a habitar apenas com a família nuclear, conseguem casas mais próximas dos locais de trabalho, algumas compram casa própria.

\section{Redes Sociais}

As redes sociais têm uma relação intrínseca com o espaço, elas configuram o próprio espaço e têm, elas mesmas, uma representação espacial. As redes de apoio constituídas por familiares (mais visíveis no caso das brasileiras e cabo-verdianas) e amigos, apesar de, em geral, não integrarem mais de dois, três elementos, desempenham uma função de suporte particularmente importante no momento da chegada. $\mathrm{O}$ apoio pode revestir-se de diversas formas: psicológico, apoio no encontrar o primeiro emprego, partilha de casa, comunicação das regras e lógicas inerentes à sociedade, familiarização com os processos burocráticos de legalização. Por vezes esperava-se contar com o apoio de pessoas que nem chegaram a aparecer e são outras pessoas que ocasionalmente se vão conhecendo que se vêm a revelar os verdadeiros pontos de apoio: pessoas que se conhecem na estação dos comboios, nos locais de trabalho e na rua.

Por vezes têm-se familiares em Portugal que nem se sabe onde vivem e que não são, de facto, elementos reais da rede. Curiosamente, os nacionais são, em muitas situações, os verdadeiros elementos da rede e muitos dos elementos das redes das mulheres são outras mulheres (amigas ou familiares), sendo também em grande número as decisões de imigrar influenciadas por outras mulheres (amigas e familiares).

A análise da relação entre género e espaços urbanos, do quotidiano das mulheres na cidade e da sua relação com essa mesma cidade tem sido negligenciada nos estudos migratórios, mas revela-se um campo de reflexão fecundo. Apesar do espaço tantas vezes traduzir e revelar as próprias assimetrias, desigualdades e hierarquias sociais, a noção de espaço é uma noção criativa, que responde às pretensões de formas de vida múltiplas, alternativas, urbanas, o espaço configura oportunidades, proporciona escolhas e desafia as hegemonias de género.

$1 \quad \mathrm{O}$ rizoma não tem princípio ou fim, está sempre no meio, entre coisas, interbeing, intermezzo. A árvore é filiação, mas o rizoma é aliança, unicamente aliança. "The tree imposes the verb 'to be' but the fabric of the rhizome is the conjunction, 'and... and... and...'. This conjunction carries enough force to shake and uproot the verb 'to be'. Where are you going? Where are you coming from? What are you heading for? These are totally useless questions. Making a clean state, starting or beginning again from ground zero, seeking a beginning or a foundation-all imply a false conception of voyage and movement (a conception that is methodical, pedagogical, initiatory, symbolic...)" (DELEUZE e GUATARRI, 1987, p.25).

\section{Referências}

BHABHA, Homi. Dissemination: Time, narrative and the margins of the modern Nation. In: BHABHA, Homi. Nation and Narration. Ed. Homi Bhabha: New York and London, 1990, p. 291-322.

BAUMAN, Zygmunt. Cultura: aventuras líquidas-modernas de uma ideia. Configurações - Revista de Sociologia, n. 3, 
p. $11-22,2007$.

BELL, Vikki. Performativity and belonging: An Introduction. Theory, Culture \& Society, v. 16, n. 2, p. 1-10, 1999.

BENJAMIN, Walter. La Tarea del Traductor. In: Ensayos Escogidos. Ed: Sur: Buenos Aires, 1967, p. 79.

BOUTANG, Pierre-André; PAMART, Michel. (Dir.) L'abécédaire de Gilles Deleuze/entrétien, Claire Parnet. Paris: Éditions Montparnasse/Sodaperage, 1995.

BRAIDOTTI, Rosa. Nomadic subjects. Nova Iorque: Columbia University Press, 1994.

CAMILLERI, Carmel. Stratégies Identitaires, Paris: PUF, 1991.

CERTEAU, Michel de. The practice of everyday life. Berkeley: University of California Press, 1984.

CHAMBERLAIN, Mary. Gender and the narratives of migration. History Workshop Journal, v. 43, p. 87 - 108, 1997.

CROSS, Malcom; KEITH, Michael. Racism, the City and the State. Londres: Routledge, 1993.

DARKE, Jane. The man-shaped city. In: BOOTH, Chris; DARKE, Jane; YEANDLE, Sue (Eds.). Changing Places: Women's Lives in the City. Londres: Paul Chapman Publishing Ltd., 1996, p. 88 - 99.

DELEUZE, Gilles; GUATTARI. Mille Plateaux, Minuit, coll. Paris: Critique, 1980.

DELEUZE, Gilles; GUATARRI, Félix. A Thousand Plateaus. Capitalism and Schizophrenia. Minneapolis: University of Minnesota Press, 1987.
EADE, John. Placing London: From Imperial City to Global City. Oxford: Berghahn Books, 2000.

FENSTER, Tovi. Ethnicity, citizenship and gender: the case of Ethiopian immigrant women is Israel. Gender, Place and Culture, v. 5, n. 2, p. 177 - 189, 1998.

FENSTER, Tovi. The right to the gendered city: different formations of belonging in everyday life. Journal of Gender Studies, v. 14, n. 3, p. 217 - 231, 2005.

FINE, Michelle. Disruptive voices: The possibilities of feminist research. Ann Arbor: University of Michigan Press, 1992.

FORTIER, Anne-Marie. Migrant belongings: Memory, space, identity. Oxford/Nova Iorque: Berg, 2000.

GARDNER, Katy. Narrating location: space, age and gender among Bengali elders in East London. Oral History. v. 27, n. 1, p. $65-74$, 1999.

GRILLO, Ralph. Plural cities in comparative perspective. Ethnic and Racial Studies, v. 23, n. 6, p. 957 - 981, 2000.

GUPTA, Akhil; FERGUSON, James. Beyond culture: Space, identity and the politics of difference. St. Leonards: Allen and Unwin, 1992.

HALL, Suart. Introduction: Who needs identity?. In: HALL, Stuart; GAY, Paul du (Eds.). Questions of Cultural Identity. London: Sage, 1996, p. 1 - 17.

HAYDEN, Dolores. The power of place. Cambridge, MA: MIT Press, 1995.

HERBERT, Joanna. Negotiating boundaries in the city: Migration, ethnicity and gender in Britain (Studies in Migration and 
Diasporas). Hampshire: Ashgate, 2007.

JACKSON, Peter; PENROSE, Jan. Constructions of Race, Place and Nation. Londres: UCL Press, 1993.

KAPLAN, Caren. Questions of travel: Postmodern discourses of displacements. Durham e Londres: Duke University Press, 1996.

KASINITZ, Philip. Caribbean New York: Black Immigrants and the Politics of Race. Ithaca: Cornell University Press, 1992.

KATZ, Cindi. Growing girls/closing circles: limits on the spaces of knowing in rural Sudan and United States cities. In: KATZ, Cindi; MONK, Janice. (Eds.). Full Circles: Geographies of Women over the Life Course. Londres: Routledge, 1993, p. 88 106.

KEITH, Michael; PILE, Steve. Place and the Politics of Identity, Londres e Nova Iorque: Routledge ,1993.

KUGELMASS, Jack. Masked Culture: The Greenwich Village Halloween Parade. Nova Iorque: Columbia University Press, 1994.

LAWRENCE, Denise. Transcendence of place: the role of La Placeta in Valencia's Las Fallas. In: PERVIN, Lawrence A. \& LEWIS, Michael. (Eds.) Perspectives in Interactional Psychology. Nova Iorque: Plenum, 1992, p. 287 - 327.

LEACH, Neil. Belonging: towards a theory of identification with space. In: Jean HILLIER; Emma ROOKSBY (Eds.). Habitus: A sense of place. Aldershot: Ashgate, 2002, p. 281 - 289.

MACLEOAD, Arlene Elowe. Accommodating protest: working women, the new veling and change in Cairo. Nova Iorque: Columbia University Press, 1991.

MASSEY, Doreen. Power-geometry and a progressive sense of place. In: BIRD, J.; CURTIS, B.; PUTNAM, T. and TICKNER, L. Mapping the futures: local cultures, global change. Eds: Routledge, London, 1993.

MASSEY, Doreen. Space, Place and Gender. Cambridge: Polity Press, 1998.

MASSEY, Doreen; DENTON, Nancy. American apartheid: Segregation and the making of the underclass. Cambridge, MA: Harvard University Press, 1993.

MILL, John Stuart. De la liberté. Trans. C. Dupont. Paris: Gallimard, 1990.

PARSONS, Deborah. Street walking the Metropolis: Women, the City and Modernity. Cambridge: Cambridge University Press, 2000.

PROBYN, Elspeth. Outside Belongings. Nova Iorque e Londres: Routledge, 1996.

PROBYN, Elspeth. Sexing the Self: Gendered positions in cultural studies. Londres: Routledge, 1993.

RYAN, Louise. Moving spaces and changing places: Irish women's memories of emigration to Britain in the 1930s'. Journal of Ethnic Studies. v. 29, n. 1, p. $67-82$, 2003.

THOMSON, Alistair. Anzac memories. In: Robert PERKS; Alister THOMSON. (Eds.).The Oral History Reader. Londres: Routledge, 1998, p. 300 - 310.

WALTON, John. A Storied Land: Community and memory in Monterey. Londres: University of California Press, 
2001.

WEKERLE,Gerda. A woman's place is in the city. Antipode, v. 16, n. 3, p. 11 - 16, 1984.

WILSON, Elizabeth. The Sphinix in the City: Urban Life, the Control of Disorder, and Women. Berkeley: University of California Press, 1991.

WOMEN and GEOGRAPHY GROUP. Feminist Geographies: Exploration in Diversity and Difference. Longman: Londres, 1997.

YOUNG, Iris Mary. Polity and group difference: a critique of the ideal of universal citizenship. In: SHAFIR, Gershon (Eds.). The Citizenship Debate. University of Minnesota Press, 1998, p. 263 - 290. 